\title{
NOUVELle
}

\section{Comment un suppresseur de tumeur se prend au jeu de la prolifération leucémique chez l'homme}

Françoise Pflumio, Sophie Kusy, Bastien Gerby, Paul-Henri Roméo
Institut de radiobiologie cellulaire et moléculaire, UMR967 Inserm/Université Paris 7 et Paris 11, CEA, 18 , route du Panorama, 92260 Fontenay-aux-Roses, France. francoise.pflumio@cea.fr
> Le développement leucémique est classiquement vu comme le résultat de l'accumulation de plusieurs anomalies génétiques qui altèrent l'expression d'un ensemble de gènes codant pour des protéines impliquées dans la différenciation, la prolifération, l'apoptose et le cycle cellulaires. Certaines de ces anomalies génétiques touchent des gènes codant des protéines qui ont un rôle essentiel dans le développement normal du tissu considéré. Les leucémies aiguës lymphoblastiques T (LAL-T) constituent une pathologie hématologique dont le pronostic reste péjoratif, surtout en cas de rechute. Ces leucémies résultent de la prolifération non régulée de progéniteurs du lignage lymphocytaire $T$, bloqués à certaines étapes de la différenciation thymique.

\section{TAL1 et LAL-T :}

une énigme pour les souris

L'expression anormale du facteur de transcription appelé stem cell leukemia (SCL) ou T-cell acute leukemia I (TALI) dans le lignage $T$ est l'anomalie la plus récurrente dans les $L A L-T$, puisque la protéine TALl est détectée dans les cellules leucémiques de 30 à $60 \%$ des patients. TALl, dont le gène a été cloné à l'origine à partir d'une translocation détectée chez un patient souffrant de LAL-T, est un régulateur-clé de l'hématopoïèse embryonnaire et adulte normale [1]. Chez l'adulte, malgré une longue controverse $[2,3]$, le rôle de TALl dans les cellules souches hématopoïétiques (CSH) a été récemment confirmé : ce facteur de transcription assurerait le maintien des CSH en préservant leur quiescence [4] et/ou en limitant leur apoptose [5]. TAL1 participe aussi à la régulation moléculaire des différenciations érythroïde, mégacaryocytaire et granulo-monocytaire [1] et il n'est pas exprimé au cours des différenciations lymphocytaires $T$ et B [6]. Sur le plan mécanistique, TALl régule la transcription de nombreux gènes codant des protéines impliquées dans la différenciation, l'apoptose ou le cycle cellulaire en participant à la formation de complexes transcriptionnels contenant des protéines telles que GATAl, 2 ou 3 , Idbl, LMOl/2 et un membre de la famille ع2A [1]. Le mécanisme d'action de TALl dans le développement leucémique $T$ est longtemps resté une énigme, en particulier parce que la surexpression de TALI dans les modèles murins de différenciation lymphocytaire $T$ ne provoquait la leucémie observée chez l'homme qu'avec une très faible pénétrance. Les protéines de la famille E2A étant nécessaires pour assurer un développement lymphocytaire normal, il a été proposé depuis longtemps que l'expression aberrante de TALl dans les cellules lymphoïdes T provoque la formation d'hétérodimères TALl/E2A. Cette interaction détournerait les protéines de la famille $\varepsilon 2 A$ de leur fonction première dans la lymphopoïèse T normale, entraînant un blocage de la différenciation et une prolifération excessive. Cette assertion est liée à des faits expérimentaux: les souris dépourvues du membre $\varepsilon 47 \mathrm{de}$ la famille $22 A$ développent des leucémies $T$ et la surexpression de la protéine Idl qui titre les membres de la famille ¿2A provoque des leucémies $T$ chez la souris $[7,8]$.
NKX3.1, la cible inattendue de TAL1 dans les LAL-T humaines

Mais ce qui est vrai chez la souris, est-il toujours vrai chez l'homme? La réponse est bien sûr non ! En utilisant des techniques de transcriptomes comparatifs entre des lignées cellulaires issues de LAL-T humaines exprimant TALl, mais modifiées génétiquement pour diminuer cette expression, nous avons mis en évidence le lien entre l'expression de TALl et l'expression des transcrits codant pour la protéine NKX3.1, connue comme un suppresseur de tumeur dans les cancers de la prostate [9]. Cette expression anormale de NKX3.1 dans les LAL-T humaines avait été préalablement observée dans une étude d'expression de transcrits à haut débit, réalisée dans une large cohorte de LAL-T exprimant TALI [10]. Par des expériences d'immunoprécipitation de la chromatine, nous avons pu établir que TALl pouvait directement contrôler l'expression de NKX3.1 en se liant à la protéine GATA3 fixée sur une région régulatrice $d u$ gène $N K X 3.1$ et en modifiant localement la structure chromatinienne de cette région régulatrice. De fait, l'interaction TALI/GATA3 sur cette région permet la formation d'un complexe transcriptionnel contenant les protéines $\mid \mathrm{dbl}$ et LMOl ou 2, complexe qui rend cette région permissive à la transcription en empêchant la liaison du facteur répresseur HIPl $\alpha$ et en diminuant les triméthylations sur lysine de l'histone H3 (Figure 1). De façon intéressante, la séquence régulatrice du gène humain NKX3.1 fixant GATA3 n'est pas conservée chez la souris, ce qui explique pourquoi 


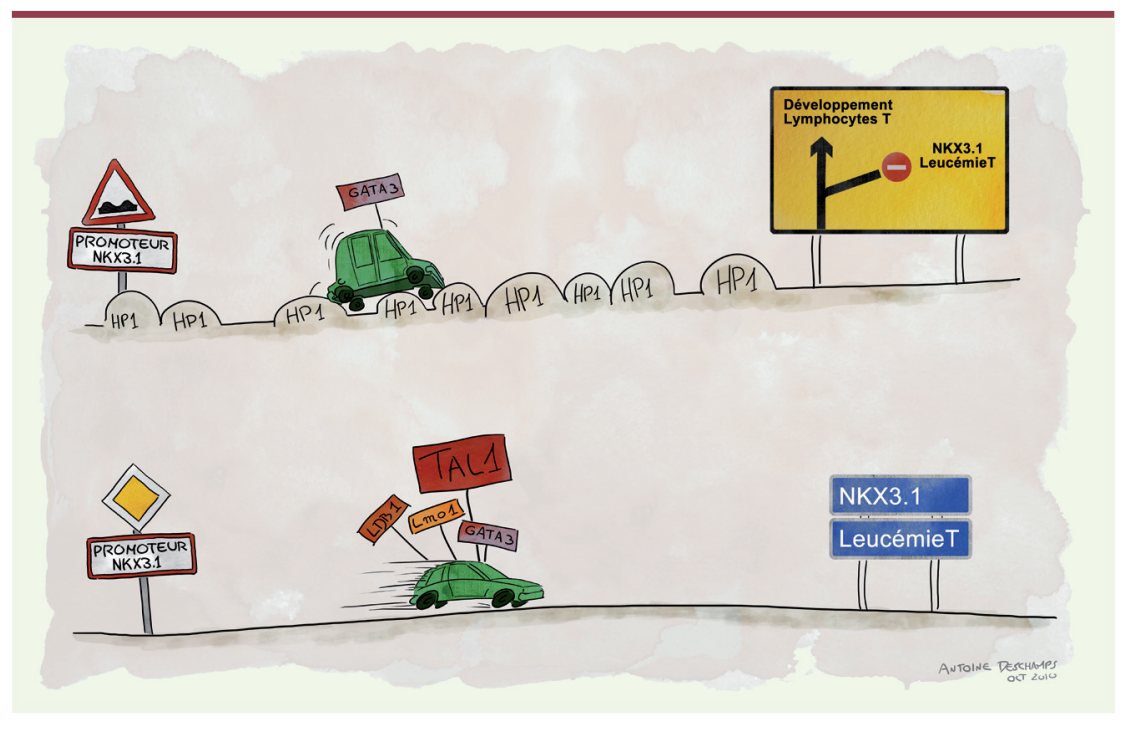

le gène NKX3.1 n'a pas d'expression anormale dans les modèles murins de LAL-T TALI dépendants et la difficulté à reproduire la leucémie T TALl dépendante chez la souris.

D'un point de vue fonctionnel, l'extinction de NKX3.1 dans plusieurs lignées leucémiques $\mathrm{T}$ exprimant TALl provoque un allongement de la durée du cycle cellulaire et conduit à une croissance fortement réduite en culture. De plus, l'inhibition de la croissance cellulaire liée à une diminution de l'expression de TALl peut être partiellement réversée par l'expression forcée de NKX3.1 dans ces cellules démontrant bien que NKX3.1 est un (mais pas le seul) intermédiaire de l'effet prolifératif de TALl. Cependant, il serait hasardeux d'extrapoler des résultats obtenus à partir de lignées cellulaires perpétuées en culture depuis des années à des cellules de patients au diagnostic. Au cours des dernières années, nous avons développé des modèles expérimentaux de culture, de greffe et de modification génique des LAL-T humaines à partir de cellules de patients $[11,12]$, modèles qui permettent à présent d'explorer des questions relatives à la régulation moléculaire $d u$ développement leucémique. En utilisant ces outils, nous avons montré la dépendance des LAL-T primaires vis-à-vis des niveaux d'expression de TALl et de NKX3.1 puisqu'une diminution drastique de l'expression de TALl ou de NKX3.1 compromet la croissance des cellules leucémiques en culture et le développement leucémique dans des souris immunodéficientes, tandis qu'une diminution partielle n'altère que peu ces caractéristiques.

\section{Conclusion}

Ce travail jette un éclairage nouveau sur une des missions impossibles de TALl dans le processus leucémique: la corruption de l'expression du gène NKX3.1 qui, réprimé par GATA3 dans la lymphopoïèse $T$, devient actif grâce à TAL1. De plus, l'originalité de NKX3.1 est son caractère oncogénique dans le lignage lymphocytaire T alors qu'il est décrit comme un suppresseur de tumeur dans la prostate, montrant bien le caractère tissu spécifique de l'action de ces facteurs de transcription. Enfin, NKX3.1 joue un rôle majeur dans la maintenance des cellules souches normales de la prostate et il sera intéressant d'étudier son rôle dans celle des cellules souches leucémiques T. La découverte du lien entre NKX3.1 et TALl va aussi permettre de caractériser les voies métaboliques dépendantes de TALl et indépendantes de NKX3.1, ce qui pourra participer à une approche intégrative des LAL-T humaines. $\diamond$

How a suppressor of tumor gets hooked of the leukaemic proliferation in man
Figure 1. Rôle de TALI dans l'expression de NKX3.1 et l'induction leucémique. Illustration réalisée par Antoine Deschamps, PDI, 1800 Seaport Boulevard, Redwood City CA, 94063, USA (aaaah_tonio@hotmail.com).

\section{CONFLIT D'INTÉRÊTS}

Les auteurs déclarent n'avoir aucun conflit d'intérêts concernant les données publiées dans cet article.

\section{RÉFÉRENCES}

1. Lecuyer $\varepsilon$, Hoang T. SCL: from the origin of hematopoiesis to stem cells and leukemia. Exp Hematol 2004 ; 32 : 11-24.

2. Mikkola HK, Klintman J, Yang $\mathrm{H}$, et al. Haematopoietic stem cells retain long-term repopulating activity and multipotency in the absence of stem-cell leukaemia SCL/tal-1 gene. Nature 2003 ; 421 : 547-51.

3. Reynaud D, Ravet $\varepsilon$, Titeux M, et al. SCL/TALI expression level regulates human hematopoietic stem cell self-renewal and engraftment. Blood 2005; $106: 2318-28$.

4. Lacombe J, Herblot S, Rojas-Sutterlin S, et al. $\mathrm{Scl}$ regulates the quiescence and the long-term competence of hematopoietic stem cells. Blood 2009; $115: 792-803$.

5. Souroullas GP, Salmon JM, Sablitzky F, Curtis DJ, Goodell MA. Adult hematopoietic stem and progenitor cells require either Lyll or Scl for survival. Cell Stem Cell $2009 ; 4: 180-6$.

6. Zhang Y, Payne KJ, Zhu Y, et al. SCL expression at critical points in human hematopoietic lineage commitment. Stem Cells $2005 ; 23$ : 852-60.

7. Bain G, Engel I, Robanus Maandag $\varepsilon C$, et al. દ2A deficiency leads to abnormalities in alphabeta T-cell development and to rapid development of T-cell lymphomas. Mol Cell Biol 1997 ; 17 : 4782-91.

8. Morrow MA, Mayer EW, Perez CA, Adlam M, Siu G. Overexpression of the Helix-Loop-Helix protein Id2 blocks T cell development at multiple stages. Mol Immunol 1999; $36: 491-503$.

9. Kusy S, Gerby B, Goardon N, et al. NKX3.1 is a direct TALl target gene that mediates proliferation of TALl-expressing human T cell acute lymphoblastic leukemia.J Exp Med 2010 ; 207 : 2141-56.

10. Soulier J, Clappier $\varepsilon$, Cayuela JM, et al. HOXA genes are included in genetic and biologic networks defining human acute T-cell leukemia (T-ALL). Blood 2005; $106: 274-86$.

11. Gerby B, Armstrong F, de la Grange PB, et al. Optimized gene transfer into human primary leukemic $T$ cell with NOD-SCID/leukemia-initiating cell activity. Leukemia $2010 ; 24: 646-9$.

12. Armstrong F, Brunet de la Grange $P$, Gerby B, et al. NOTCH is a key regulator of human T-cell acute leukemia initiating cell activity. Blood 2009; $113: 1730-40$. 


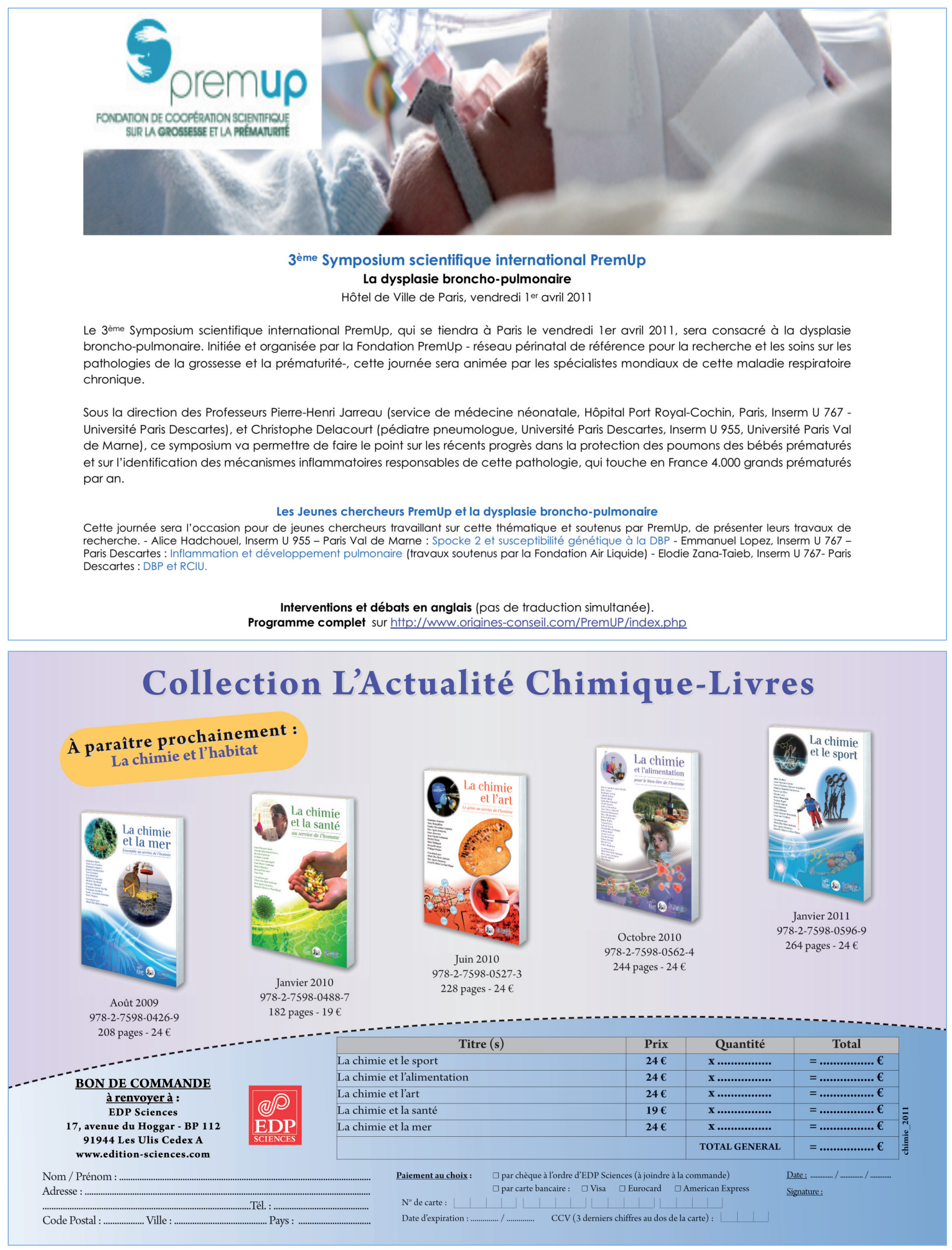

